\title{
Spinning Kerr black holes with stationary massive scalar clouds: the large-coupling regime
}

\author{
Shahar Hod \\ Marine sciences, The Ruppin Academic Center, \\ Ruppin, Emeq Hefer 40250, Israel \\ Biotechnology, The Hadassah Academic College, \\ 37 Hanevi'im St., Jerusalem 9101001, Israel \\ E-mail: shaharhod@gmail.com
}

ABSTRACT: We study analytically the Klein-Gordon wave equation for stationary massive scalar fields linearly coupled to spinning Kerr black holes. In particular, using the WKB approximation, we derive a compact formula for the discrete spectrum of scalar field masses which characterize the stationary composed Kerr-black-hole-massive-scalar-field configurations in the large-coupling regime $M \mu \gg 1$ (here $M$ and $\mu$ are respectively the mass of the central black hole and the proper mass of the scalar field). We confirm our analytically derived formula for the Kerr-scalar-field mass spectrum with numerical data that recently appeared in the literature.

Keywords: Black Holes, Classical Theories of Gravity

ARXIV EPRINT: 1612.00014 


\section{Contents}

1 Introduction 1

2 Description of the system 3

3 The resonance mass spectrum of the composed Kerr-black-hole-massive$\begin{array}{ll}\text { scalar-field configurations } & 4\end{array}$

$\begin{array}{lll}4 & \text { Numerical confirmation } & 7\end{array}$

$\begin{array}{lll}5 & \text { Summary } & 7\end{array}$

\section{Introduction}

The complex interactions between fundamental matter fields and general relativistic black holes have been the focus of intense research efforts during the last five decades (see [1-95] and references therein). Especially interesting are the physical properties of astrophysically realistic spinning black holes coupled to bosonic matter fields. As explicitly demonstrated recently [8-35], these integer-spin fields can form non-trivial spatially regular stationary matter configurations in rotating curved black-hole spacetimes.

In particular, recent analytical [8-13] and numerical [14-35] studies of the stationary sector of the coupled Einstein-Klein-Gordon field equations have revealed the intriguing fact that, for a given spinning black hole of mass $M$ and angular momentum per unit mass $a$, there exists a discrete spectrum of resonant field masses, $\{M \mu(a / M, m ; n)\}_{n=0}^{n=\infty}, 1,2,3,4$ which characterize a family of stationary spatially regular scalar configurations that can be supported in the external region of the corresponding rotating black-hole spacetime.

Interestingly, these stationary bound-state bosonic matter configurations are characterized by proper azimuthal frequencies which are in resonance with (that is, are multiple integers of) the angular frequency of the supporting central black hole [8-35]: ${ }^{5}$

$$
\omega_{\text {field }}=\omega_{\mathrm{c}} \equiv m \Omega_{\mathrm{H}},
$$

\footnotetext{
${ }^{1}$ We shall use natural units in which $G=c=\hbar=1$.

${ }^{2}$ Note that the proper mass $\mu$, which characterizes the scalar field mode, stands for $\mu / \hbar$. Hence, this physical parameter of the matter field has the dimensions of (length) ${ }^{-1}$.

${ }^{3}$ Here the dimensionless integer parameters $m$ and $n$ are respectively the azimuthal harmonic index and the resonance parameter of the stationary bound-state massive scalar field mode [see eqs. (2.5) and (3.7) below].

${ }^{4}$ We shall assume $a>0$ and $m>0$ for the black-hole-field physical parameters without loss of generality.

${ }^{5}$ It is worth noting that, for a given value of the azimuthal harmonic index $m$ of the scalar mode, the characteristic proper frequency $\omega_{\text {field }}=m \Omega_{\mathrm{H}}$ of the stationary field configuration coincides with the threshold (critical) frequency $\omega_{\mathrm{c}}$ for the superradiant scattering phenomenon of bosonic fields in the spinning Kerr black-hole spacetime [2-6].
} 
where the integer $m$ is the azimuthal harmonic index of the scalar mode and $[1,96]^{6}$

$$
\Omega_{\mathrm{H}}=\frac{a}{r_{+}^{2}+a^{2}}
$$

is the angular velocity which characterizes the outer horizon of the spinning Kerr black hole.

For a scalar field of proper mass $\mu$, the resonant field frequency (1.1) which, for a given value of the azimuthal harmonic index $m$, characterizes a stationary bound-state scalar configuration in the Kerr black-hole spacetime, is also bounded from above by the inequality [8-35]

$$
\omega_{\text {field }}^{2}<\mu^{2} .
$$

The characteristic relation (1.3) guarantees that the massive scalar configuration is spatially bounded to the black hole. In particular, low frequency stationary scalar modes in the regime (1.3) are captured inside an effective potential well which characterizes the composed black-hole-massive-scalar-field system [see eq. (3.5) below]. Thus, matter configurations made of massive scalar fields in the regime (1.3) cannot radiate their energies to asymptotic infinity $[8-35] .^{7}$

The discrete spectrum of resonant field masses $\{M \mu(a / M, m)\}$, which characterizes the stationary bound-state scalar configurations in the spinning Kerr black-hole spacetime, has been computed numerically in [14-35]. For rapidly-rotating (near-extremal) black holes in the regime $a / M \rightarrow 1$, the characteristic mass spectrum of the composed blackhole-linearized-massive-scalar-field configurations has been studied analytically in [8-13]. To the best of our knowledge, the characteristic resonant spectrum $\{M \mu(a / M, m)\}$ of the stationary bound-state massive scalar configurations has not been determined analytically thus far for generic $^{8}$ values $a / M \in(0,1)$ of the dimensionless rotation parameter which characterizes the central supporting black holes.

The main goal of the present paper is to derive a compact analytical formula for the discrete spectrum $\{M \mu(a / M, m)\}$ of field masses which characterize the stationary bound-state scalar configurations in generic ${ }^{8}$ Kerr black-hole spacetimes. To this end, we shall study analytically the Klein-Gordon wave equation for stationary massive scalar fields linearly coupled to rotating Kerr black-hole spacetimes. In particular, using the WKB approximation, we shall derive below a compact analytical formula for the discrete spectrum $\{M \mu(a / M, m)\}$ of scalar field masses which characterize the stationary boundstate Kerr-black-hole-massive-scalar-field configurations in the eikonal large-mass $M \mu \gg 1$

\footnotetext{
${ }^{6}$ Here $r_{+}$is the radius of the outer black-hole horizon [see eq. (2.3) below].

${ }^{7}$ It is worth noting that the stationary bound-state scalar configurations, which are characterized by the resonant oscillation frequencies (1.1), divide the parameter space of the composed black-hole-scalarfield system into two physically distinct regimes [2-95]. In particular, for a given value of the azimuthal harmonic index $m$, massive bosonic field configurations in the regime $\omega_{\text {field }}<\omega_{\mathrm{c}}$ are known to develop exponentially growing superradiant instabilities [provided that they also conform to the inequality (1.3) which characterizes the bound-state configurations in the black-hole spacetime], whereas field configurations in the regime $\omega_{\text {field }}>\omega_{\mathrm{c}}$ are known to decay in time [2-95].

${ }^{8}$ We use here the term 'generic Kerr black holes' to describe spinning black holes that belong to the entire physical range $a / M \in(0,1)$ of the rotation parameter (that is, spinning black holes which are not necessarily near-extremal ones).
} 
regime. Most importantly, this analytically derived formula [see eq. (3.14) below] for the scalar resonance spectrum would be valid in the entire physical range $a / M \in(0,1)$ of the black-hole rotation parameter.

\section{Description of the system}

We shall analyze the properties of a physical system which is composed of a stationary scalar field $\Psi$ of mass $\mu$ and proper frequency $\omega_{\text {field }}=m \Omega_{\mathrm{H}}$ [see eqs. (1.1) and (1.2)] which is linearly coupled to a central rotating Kerr black hole of mass $M$ and angular momentum per unit mass $a$. Using the Boyer-Lindquist coordinates $(t, r, \theta, \phi)$, one can describe the spinning black-hole spacetime by the line element $[1,96]$

$$
d s^{2}=-\frac{\Delta}{\rho^{2}}\left(d t-a \sin ^{2} \theta d \phi\right)^{2}+\frac{\rho^{2}}{\Delta} d r^{2}+\rho^{2} d \theta^{2}+\frac{\sin ^{2} \theta}{\rho^{2}}\left[a d t-\left(r^{2}+a^{2}\right) d \phi\right]^{2},
$$

where

$$
\Delta \equiv r^{2}-2 M r+a^{2} ; \quad \rho^{2} \equiv r^{2}+a^{2} \cos ^{2} \theta
$$

The zeros of $\Delta$,

$$
r_{ \pm}=M \pm \sqrt{M^{2}-a^{2}}
$$

determine the horizon radii of the Kerr black-hole spacetime.

The spatial and temporal properties of a linearized massive scalar field in the curved black-hole spacetime are determined by the familiar Klein-Gordon wave equation

$$
\left(\nabla^{\nu} \nabla_{\nu}-\mu^{2}\right) \Psi=0
$$

Substituting the metric components of the Kerr black-hole line element (2.1) into the Klein-Gordon wave equation (2.4) and using the scalar field decomposition ${ }^{9}$

$$
\Psi(t, r ; \omega, \theta, \phi)=\sum_{l, m} e^{i m \phi} S_{l m}\left(\theta ; m, a \sqrt{\mu^{2}-\omega^{2}}\right) R_{l m}(r ; M, a, \mu, \omega) e^{-i \omega t},
$$

one obtains the characteristic ordinary differential equation (known as the spheroidal angular equation) [97-112]

$$
\frac{1}{\sin \theta} \frac{d}{d \theta}\left(\sin \theta \frac{d S_{l m}}{d \theta}\right)+\left[K_{l m}+a^{2}\left(\mu^{2}-\omega^{2}\right) \sin ^{2} \theta-\frac{m^{2}}{\sin ^{2} \theta}\right] S_{l m}=0
$$

for the angular part $S_{l m}$ of the scalar eigenfunction $\Psi .{ }^{10}$ Spheroidal harmonic eigenfunctions $\left\{S_{l m}\right\}$ which are regular at the angular poles $\theta=0$ and $\theta=\pi$ are known to be characterized by a discrete set $\left\{K_{l m}\right\}$ of angular eigenvalues [97-112]. In particular, it has recently been proved that, in the eikonal $(|m| \gg 1)$ regime, the angular eigenvalues are

\footnotetext{
${ }^{9}$ Here the integer $l \geq|m|$ is the spheroidal harmonic index of the bound-state scalar field mode.

${ }^{10}$ The angular eigenfunctions $\left\{S_{l m}\right\}$ are the familiar spheroidal harmonics.
} 
given by the asymptotic large- $m$ analytical formula $[112]^{11,12}$

$$
K_{l m}=m^{2}-a^{2}\left(\mu^{2}-\omega^{2}\right)+[2(l-|m|)+1] \sqrt{m^{2}+a^{2}\left(\mu^{2}-\omega^{2}\right)}+O(1) .
$$

Substituting the scalar field decomposition (2.5) into (2.4), and using the line element (2.1) which characterizes the spinning Kerr black-hole spacetime, one finds the characteristic ordinary differential equation [97-99]

$$
\Delta \frac{d}{d r}\left(\Delta \frac{d R_{l m}}{d r}\right)+\left\{\left[\omega\left(r^{2}+a^{2}\right)-m a\right]^{2}+\Delta\left[2 m a \omega-\mu^{2}\left(r^{2}+a^{2}\right)-K_{l m}\right]\right\} R_{l m}=0
$$

for the radial part $R_{l m}$ of the scalar eigenfunction $\Psi$, where the angular eigenvalues $\left\{K_{l m}\right\}$ that explicitly appear in the radial equation (2.8) should be determined from the spheroidal differential equation (2.6) [see, in particular, the asymptotic angular spectrum (2.7) [112]]. Physically acceptable solutions of the scalar radial equation (2.8) are characterized by purely ingoing waves (as measured by a comoving observer) at the black-hole outer horizon [8-72]:

$$
R_{l m} \sim e^{-i\left(\omega-m \Omega_{\mathrm{H}}\right) y} \quad \text { for } \quad r \rightarrow r_{+} \quad(y \rightarrow-\infty),
$$

where the radial coordinate $y$, to be used below, is defined by the differential relation $d y=$ $\left(r^{2} / \Delta\right) d r$ [see eq. (3.2) below]. In addition, the spatially regular bound-state (finite-mass) configurations of the massive scalar fields in the black-hole spacetime are characterized by asymptotically decaying radial eigenfunctions at spatial infinity [8-72]:

$$
R_{l m} \sim \frac{1}{r} e^{-\sqrt{\mu^{2}-\omega^{2}} r} \quad \text { for } \quad r \rightarrow \infty \quad(y \rightarrow \infty) .
$$

The physically motivated boundary conditions (2.9) and (2.10) of the radial scalar equation (2.8), together with the resonance condition (1.1), determine the discrete spectrum $\{M \mu(m, a / M ; n)\}$ of scalar field masses which characterize the stationary composed Kerrblack-hole-linearized-massive-scalar-field configurations. In the next section we shall use analytical techniques in order to determine the characteristic Kerr-scalar-field resonance spectrum in the large mass $(M \mu \gg 1)$ regime.

\section{The resonance mass spectrum of the composed Kerr-black-hole- massive-scalar-field configurations}

In the present section we shall derive a compact analytical formula for the discrete spectrum $\{M \mu(m, a / M ; n)\}$ of scalar field masses which characterize the composed stationary Kerrblack-hole-massive-scalar-field configurations in the eikonal large-mass regime. The trick is

\footnotetext{
${ }^{11}$ As explicitly proved in [112], the large- $m$ asymptotic formula (2.7) is valid in the regime $-a^{2}\left(\mu^{2}-\omega^{2}\right)<$ $m^{2}$. Taking cognizance of the inequality $\omega^{2}<\mu^{2}$ which characterizes the bound-state massive scalar resonances in the black-hole spacetime [see (1.3)], one finds that the requirement $-a^{2}\left(\mu^{2}-\omega^{2}\right)<m^{2}$ is trivially satisfied.

${ }^{12}$ Note that, as explicitly proved in [112], the large- $m$ analytical formula (2.7) is valid in the $l-|m| \ll$ $\sqrt{m^{2}+a^{2}\left(\mu^{2}-\omega^{2}\right)}$ regime. Substituting $k \equiv l-m \ll m$ in $(2.7)$ for the $a=0$ case, one finds $K_{l m}(a=$ $0)=l(l+1)-k(k+1)+O(1)$. Taking cognizance of the well known fact that $K_{l m}(a=0)=l(l+1)$, one can infer that, in the $a \rightarrow 0$ limit, the sub-leading $O(1)$ term in eq. (2.7) (which has not been calculated in [112]) is given by $k(k+1)$.
} 
to transform the radial differential equation (2.8) of the scalar field into the familiar form of a Schrödinger-like radial differential equation and then to perform a standard WKB analysis.

Defining the new radial eigenfunction

$$
\psi=r R
$$

and using the coordinate transformation ${ }^{13}$

$$
d y=\frac{r^{2}}{\Delta} d r
$$

one obtains from (2.8) the Schrödinger-like ordinary differential equation

$$
\frac{d^{2} \psi}{d y^{2}}-V(y) \psi=0
$$

with the effective radial potential

$V=V(r ; \omega, M, a, \mu, l, m)=\frac{2 \Delta}{r^{6}}\left(M r-a^{2}\right)+\frac{\Delta}{r^{4}}\left[K_{l m}-2 m a \omega+\mu^{2}\left(r^{2}+a^{2}\right)\right]-\frac{1}{r^{4}}\left[\omega\left(r^{2}+a^{2}\right)-m a\right]^{2}$.

Substituting into (3.4) the resonant oscillation frequency (1.1), which characterizes the stationary massive scalar field configurations in the spinning Kerr black-hole spacetime, and using the asymptotic large- $m$ analytical formula (2.7) for the characteristic eigenvalues of the angular differential equation (2.6), one finds the expression

$$
\begin{array}{r}
V(r)=m^{2} \cdot\left\{\frac{r-r_{+}}{r^{3}\left(r_{+}^{2}+a^{2}\right)^{2}}\left[\beta a^{2} r^{2}-a^{2}\left(2 M+\beta r_{-}\right) r+2 M r_{+}^{3}\right]\right. \\
\left.+\frac{1}{m} \cdot \frac{\Delta}{r^{4}}(2 k+1) \sqrt{1+\beta \frac{a^{4}}{\left(r_{+}^{2}+a^{2}\right)^{2}}}+O\left(m^{-2}\right)\right\}
\end{array}
$$

for the effective radial potential which characterizes the composed stationary Kerr-blackhole-massive-scalar-field configurations in the eikonal $m \gg 1$ regime, ${ }^{14}$ where $k \equiv l-m \ll$ $m^{12}$. Here we have used the dimensionless physical parameter $\beta$ which relates the proper mass of the stationary scalar field to its resonant oscillation frequency: ${ }^{15}$

$$
\mu^{2}=(1+\beta) \cdot \omega_{\mathrm{c}}^{2}
$$

As we shall now show, the characteristic Schrödinger-like differential equation (3.3) is amenable to a standard WKB analysis [113-117]. In particular, one finds that the effective radial potential (3.5) of the stationary composed Kerr-massive-scalar-field configurations is characterized by the presence of a binding potential well outside the black-hole

\footnotetext{
${ }^{13}$ It is worth noting that the radial range $r \in\left[r_{+}, \infty\right]$ is mapped into the radial range $y \in[-\infty,+\infty]$ by the radial differential relation $(3.2)$.

${ }^{14}$ Note that the eikonal large- $m$ regime also corresponds to the large-mass $M \mu \gg 1$ regime [see eq. (3.14) below].

${ }^{15}$ Note that the characteristic inequality (1.3) for the bound-state resonances of the massive scalar fields in the black-hole spacetime implies that $\beta>0$.
} 
horizon [118]. As shown in $[113,114,117]$, the WKB resonance condition which characterizes the bound-state resonances of the standard Schrödinger-like ordinary differential equation (3.3) in the eikonal large- $m$ regime is given by

$$
\frac{V_{\min }}{\sqrt{2 V_{\min }^{\prime \prime}}}=-\left(n+\frac{1}{2}\right) ; \quad n=0,1,2, \ldots,
$$

where a prime denotes a spatial derivative of the effective radial potential (3.5) with respect to the radial coordinate $y$ [see eq. (3.2)] of the Schrödinger-like differential equation. The subscript "min" in (3.7) means that the effective radial potential and its spatial derivatives are evaluated at the radial coordinate $r^{\text {min }}$ which characterizes the minimum point of the effective binding potential (3.5). ${ }^{16}$

The WKB resonance equation (3.7) with the (rather cumbersome) radial potential (3.5) can be solved analytically using an iteration scheme (that is, using an expansion in inverse powers of $m$ ). Keeping only the leading order $O\left(m^{2}\right)$ term of the effective radial potential (3.5), one finds from (3.7) the leading-order solution

$$
r_{0}^{\min }=r_{+} \cdot \frac{\gamma\left[1-\sqrt{1-\gamma^{2}}\right]}{2-\gamma^{2}-2 \sqrt{1-\gamma^{2}}}
$$

with

$$
\beta_{0}=\frac{(1+\gamma)\left(2-\gamma^{2}-2 \sqrt{1-\gamma^{2}}\right)}{\gamma^{3}},
$$

where

$$
\gamma \equiv \frac{r_{-}}{r_{+}}
$$

is the dimensionless ratio between the horizon radii [see eq. (2.3)] of the rotating Kerr black-hole spacetime.

Next, substituting

$$
r^{\min }=r_{0}^{\min } \cdot\left[1+\frac{r_{1}}{m}+O\left(m^{-2}\right)\right]
$$

and

$$
\beta=\beta_{0} \cdot\left[1+\frac{\beta_{1}}{m}+O\left(m^{-2}\right)\right],
$$

and keeping terms of orders $O\left(m^{2}\right)$ and $O(m)$ in the effective radial potential (3.5), one finds from (3.7) after some lengthy algebra ${ }^{17}$

$$
\begin{aligned}
\beta_{1}= & -\frac{\left(2-\gamma^{2}-2 \sqrt{1-\gamma^{2}}\right) \sqrt{1+\frac{1}{\gamma}}}{\left(1-\sqrt{1-\gamma^{2}}\right)^{2}} \\
& \cdot\left[\sqrt{-1+\gamma+\sqrt{1-\gamma^{2}}} \cdot(1+2 n)+\sqrt{2+\gamma-2 \sqrt{1-\gamma^{2}}} \cdot(1+2 k)\right] .
\end{aligned}
$$

\footnotetext{
${ }^{16}$ That is, the radial coordinate $r^{\min }$ is defined by the relation $V^{\prime}\left[r=r^{\min }(y)\right]=0$ with $V^{\prime \prime}\left[r=r^{\min }(y)\right]>0$.

${ }^{17}$ The explicit expression of the dimensionless expansion coefficient $r_{1}$ is rather cumbersome and is not important for our purposes.
} 
Finally, taking cognizance of eqs. (1.1), (1.2), (3.6), (3.9), (3.10), (3.12), and (3.13), one finds the analytical formula

$$
M \mu=m \cdot\left[\Gamma_{0}+\frac{\Gamma_{1}}{m}+O\left(m^{-2}\right)\right]
$$

for the discrete spectrum of scalar field masses which characterize the stationary composed Kerr-black-hole-massive-scalar-field configurations in the eikonal large-mass $(M \mu \gg 1)$ regime, where

$$
\Gamma_{0}=\frac{\sqrt{2(1+\gamma)\left(1-\sqrt{1-\gamma^{2}}\right)-\gamma^{2}}}{2 \gamma}
$$

and $^{18}$

$$
\Gamma_{1}=-\frac{\left(1+\frac{1}{\gamma}\right)^{3 / 2}\left(2-\gamma^{2}-2 \sqrt{1-\gamma^{2}}\right)^{2}\left[\sqrt{-1+\gamma+\sqrt{1-\gamma^{2}}} \cdot(1+2 n)+\sqrt{2+\gamma-2 \sqrt{1-\gamma^{2}}} \cdot(1+2 k)\right]}{4\left(1-\sqrt{1-\gamma^{2}}\right)^{2} \sqrt{2(1+\gamma)\left(1-\sqrt{1-\gamma^{2}}\right)-\gamma^{2}}} .
$$

\section{Numerical confirmation}

It is of physical interest to test the accuracy of the analytically derived formula (3.14) for the discrete mass spectrum of the composed stationary Kerr-black-hole-massive-scalar-field configurations. The corresponding scalar field masses of the stationary configurations were computed numerically in the very interesting work of Herdeiro and Radu [14-35]. In table 1 we display the dimensionless ratio $\mu_{\text {numerical }} / \mu_{\mathrm{wkb}}$ for the fundamental $(n=0)$ resonant mode with $l=m=10$, where $\left\{\mu_{\text {numerical }}(a / M)\right\}$ are the numerically computed [14-35] field masses of the stationary scalar configurations and $\left\{\mu_{\mathrm{wkb}}(a / M)\right\}$ are the analytically derived masses of the stationary bound-state scalar fields as given by the WKB expression (3.14). From the data presented in table 1 one finds that the agreement between the numerical data [14-35] and the analytically derived formula (3.14) for the resonant masses of the stationary black-hole-scalar-field configurations is generally better than $1 \%{ }^{19}$

\section{Summary}

The intriguing physical mechanism of rotational superradiance [2-6] allows spinning Kerr black holes to support stationary bound-state matter configurations made of massive scalar fields in their exterior regions. The physical properties of these stationary composed blackhole-field configurations have been studied extensively in recent years [8-35]. Interestingly, it has been shown [8-35] that, for a given set $\{M, a, m\}$ of the black-hole-field physical parameters, there exists a discrete spectrum $\{M \mu(a / M, m ; n)\}$ of field masses which characterize the composed stationary Kerr-black-hole-massive-scalar-field configurations. In

\footnotetext{
${ }^{18}$ Note that $\Gamma_{1}<0$, which implies $M \mu<m \Gamma_{0}$ [see eq. (3.14)] in the asymptotic $m \rightarrow \infty$ regime [118].

${ }^{19}$ It is worth mentioning that, for the particular case of rapidly-rotating (near-extremal) black holes, one can use an alternative mass formula for the stationary bound-state scalar fields (see [8-13] for details).
} 


\begin{tabular}{|c|c|}
\hline$s \equiv a / M$ & $\frac{\mu(l=m=10)}{\mu_{\mathrm{wkb}}}$ \\
\hline 0.1 & 0.99946 \\
0.2 & 0.99993 \\
0.3 & 1.00009 \\
0.4 & 1.00017 \\
0.5 & 0.99974 \\
0.6 & 1.00037 \\
0.7 & 1.00076 \\
0.8 & 1.00134 \\
0.9 & 1.00266 \\
0.95 & 1.00399 \\
0.99 & 1.00750 \\
0.999 & 1.01219 \\
\hline
\end{tabular}

Table 1. Stationary massive scalar clouds linearly coupled to spinning Kerr black holes. We display the dimensionless ratio $\mu_{\text {numerical }} / \mu_{\mathrm{wkb}}$, where $\left\{\mu_{\text {numerical }}(a / M)\right\}$ are the numerically computed [1435] field masses of the stationary scalar fields and $\left\{\mu_{\mathrm{wkb}}(a / M)\right\}$ are the analytically derived scalar field masses as given by the WKB expression (3.14). The data presented are for the fundamental $(n=0)$ resonant mode with $l=m=10$. One finds that the agreement between the numerical data and the analytical formula (3.14) is generally better than $1 \%$.

particular, for near-extremal (rapidly-rotating) Kerr black holes, the characteristic discrete mass spectrum of the composed black-hole-linearized-massive-scalar-field configurations has been studied analytically in [8-13].

In the present paper we have studied analytically the Klein-Gordon wave equation for stationary massive scalar fields linearly coupled to generic ${ }^{8}$ spinning Kerr black holes. In particular, using the WKB approximation, we have derived an explicit analytical formula [see eqs. (3.14)-(3.16)] for the discrete spectrum of scalar field masses which characterize the stationary composed Kerr-black-hole-massive-scalar-field configurations in the eikonal large-mass $M \mu \gg 1$ regime. Interestingly, the analytically derived formula (3.14) for the discrete mass spectrum of the composed Kerr-scalar-field configurations was shown to agree remarkably well with direct numerical computations [14-35] of the corresponding blackhole-massive-scalar-field resonances.

Finally, it is worth noting that, to the best of our knowledge, the analytically derived expression (3.14), which is valid in the entire physical range $a / M \in(0,1)$ of the black-hole rotation parameter, ${ }^{20}$ provides the first analytical formula for the discrete mass spectrum of the stationary bound-state linearized massive scalar field configurations in the spacetimes of generic $^{8}$ spinning Kerr black holes.

\footnotetext{
${ }^{20}$ It is worth emphasizing again that, as we explicitly shown in this paper (see the data presented in table 1), the analytical formula (3.14) is not only valid in the entire physical regime $a / M \in(0,1)$ of the supporting black hole, but it also agrees remarkably well with the exact (numerically computed [14-35]) proper masses which characterize the stationary bound-state scalar fields in the spinning Kerr black-hole spacetime.
} 


\section{Acknowledgments}

This research is supported by the Carmel Science Foundation. I would like to thank C. A. R. Herdeiro and E. Radu for sharing with me their numerical data. I thank Yael Oren, Arbel M. Ongo, Ayelet B. Lata, and Alona B. Tea for stimulating discussions.

Open Access. This article is distributed under the terms of the Creative Commons Attribution License (CC-BY 4.0), which permits any use, distribution and reproduction in any medium, provided the original author(s) and source are credited.

\section{References}

[1] S. Chandrasekhar, The mathematical theory of black holes, Oxford University Press, New York U.S.A. (1983) [inSPIRE].

[2] Ya.B. Zel'dovich, Generation of waves by a rotating body, Pis'ma Zh. Eksp. Teor. Fiz. 14 (1971) 270 [JETP Lett. 14 (1971) 180].

[3] Ya.B. Zel'dovich, Amplification of cylindrical electromagnetic waves reflected from a rotating body, Zh. Eksp. Teor. Fiz. 62 (1972) 2076 [Sov. Phys. JETP 35 (1972) 1085].

[4] A. Vilenkin, Exponential amplification of waves in the gravitational field of ultrarelativistic rotating body, Phys. Lett. B 78 (1978) 301 [INSPIRE].

[5] W.H. Press and S.A. Teukolsky, Floating orbits, superradiant scattering and the black-hole bomb, Nature 238 (1972) 211 [INSPIRE].

[6] W.H. Press and S.A. Teukolsky, Perturbations of a rotating black hole. II. Dynamical stability of the Kerr metric, Astrophys. J. 185 (1973) 649 [INSPIRE].

[7] J.D. Bekenstein, Extraction of energy and charge from a black hole, Phys. Rev. D 7 (1973) 949 [InSPIRE].

[8] S. Hod, Stationary scalar clouds around rotating black holes, Phys. Rev. D 86 (2012) 104026 [Erratum ibid. D 86 (2012) 129902] [arXiv:1211.3202] [INSPIRE].

[9] S. Hod, Stationary resonances of rapidly-rotating Kerr black holes, Eur. Phys. J. C 73 (2013) 2378 [arXiv:1311.5298] [InSPIRE].

[10] S. Hod, Kerr-Newman black holes with stationary charged scalar clouds, Phys. Rev. D 90 (2014) 024051 [arXiv:1406.1179] [InSPIRE].

[11] S. Hod, Extremal Kerr-Newman black holes with extremely short charged scalar hair, Phys. Lett. B 751 (2015) 177 [INSPIRE].

[12] S. Hod, The large-mass limit of cloudy black holes, Class. Quant. Grav. 32 (2015) 134002 [arXiv:1607.00003] [INSPIRE].

[13] S. Hod, A no-short scalar hair theorem for rotating Kerr black holes, Class. Quant. Grav. 33 (2016) 114001 [INSPIRE].

[14] C.A.R. Herdeiro and E. Radu, Kerr black holes with scalar hair, Phys. Rev. Lett. 112 (2014) 221101 [arXiv: 1403.2757] [INSPIRE].

[15] C. Herdeiro and E. Radu, Ergosurfaces for Kerr black holes with scalar hair, Phys. Rev. D 89 (2014) 124018 [arXiv:1406.1225] [InSPIRE]. 
[16] C.A.R. Herdeiro and E. Radu, A new spin on black hole hair, Int. J. Mod. Phys. D 23 (2014) 1442014 [arXiv:1405.3696] [InSPIRE].

[17] Y. Brihaye, C. Herdeiro and E. Radu, Myers-Perry black holes with scalar hair and a mass gap, Phys. Lett. B 739 (2014) 1 [arXiv: 1408.5581] [InSPIRE].

[18] C.L. Benone, L.C.B. Crispino, C. Herdeiro and E. Radu, Kerr-Newman scalar clouds, Phys. Rev. D 90 (2014) 104024 [arXiv:1409.1593] [InSPIRE].

[19] J.C. Degollado and C.A.R. Herdeiro, Wiggly tails: a gravitational wave signature of massive fields around black holes, Phys. Rev. D 90 (2014) 065019 [arXiv: 1408.2589] [INSPIRE].

[20] C. Herdeiro, E. Radu and H. Runarsson, Non-linear Q-clouds around Kerr black holes, Phys. Lett. B 739 (2014) 302 [arXiv:1409.2877] [InSPIRE].

[21] C. Herdeiro and E. Radu, Construction and physical properties of Kerr black holes with scalar hair, Class. Quant. Grav. 32 (2015) 144001 [arXiv:1501.04319] [InSPIRE].

[22] C.A.R. Herdeiro and E. Radu, Asymptotically flat black holes with scalar hair: a review, Int. J. Mod. Phys. D 24 (2015) 1542014 [arXiv: 1504.08209] [InSPIRE].

[23] C.A.R. Herdeiro and E. Radu, How fast can a black hole rotate?, Int. J. Mod. Phys. D 24 (2015) 1544022 [arXiv:1505.04189] [InSPIRE].

[24] J.C. Degollado and C.A.R. Herdeiro, Stationary scalar configurations around extremal charged black holes, Gen. Rel. Grav. 45 (2013) 2483 [arXiv:1303.2392] [INSPIRE].

[25] P.V.P. Cunha, C.A.R. Herdeiro, E. Radu and H.F. Runarsson, Shadows of Kerr black holes with scalar hair, Phys. Rev. Lett. 115 (2015) 211102 [arXiv:1509.00021] [INSPIRE].

[26] B. Kleihaus, J. Kunz and S. Yazadjiev, Scalarized hairy black holes, Phys. Lett. B 744 (2015) 406 [arXiv: 1503.01672] [INSPIRE].

[27] C.A.R. Herdeiro, E. Radu and H. Rúnarsson, Kerr black holes with self-interacting scalar hair: hairier but not heavier, Phys. Rev. D 92 (2015) 084059 [arXiv:1509. 02923] [INSPIRE].

[28] C. Herdeiro, J. Kunz, E. Radu and B. Subagyo, Myers-Perry black holes with scalar hair and a mass gap: unequal spins, Phys. Lett. B 748 (2015) 30 [arXiv:1505.02407] [INSPIRE].

[29] C. Herdeiro, E. Radu and H. Runarsson, Kerr black holes with Proca hair, Class. Quant. Grav. 33 (2016) 154001 [arXiv:1603.02687] [INSPIRE].

[30] C.A.R. Herdeiro, E. Radu and H.F. Rúnarsson, Spinning boson stars and Kerr black holes with scalar hair: the effect of self-interactions, Int. J. Mod. Phys. D 25 (2016) 1641014 [arXiv: 1604.06202] [INSPIRE].

[31] Y. Brihaye, C. Herdeiro and E. Radu, Inside black holes with synchronized hair, Phys. Lett. B 760 (2016) 279 [arXiv:1605.08901] [InSPIRE].

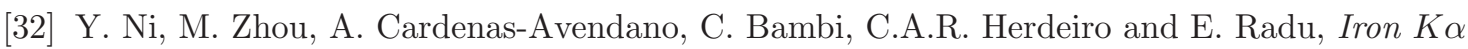
line of Kerr black holes with scalar hair, JCAP 07 (2016) 049 [arXiv:1606.04654] [INSPIRE].

[33] M. Wang, Quantum and classical aspects of scalar and vector fields around black holes, arXiv: 1606.00811 [INSPIRE]. 
[34] J.F.M. Delgado, C.A.R. Herdeiro and E. Radu, Violations of the Kerr and Reissner-Nordström bounds: horizon versus asymptotic quantities,

Phys. Rev. D 94 (2016) 024006 [arXiv:1606.07900] [InSPIRE].

[35] J.F.M. Delgado, C.A.R. Herdeiro, E. Radu and H. Runarsson, Kerr-Newman black holes with scalar hair, Phys. Lett. B 761 (2016) 234 [arXiv:1608.00631] [INSPIRE].

[36] T. Damour, N. Deruelle and R. Ruffini, On quantum resonances in stationary geometries, Lett. Nuovo Cim. 15 (1976) 257 [INSPIRE].

[37] S.L. Detweiler, Klein-Gordon equation and rotating black holes, Phys. Rev. D 22 (1980) 2323 [INSPIRE].

[38] H. Furuhashi and Y. Nambu, Instability of massive scalar fields in Kerr-Newman space-time, Prog. Theor. Phys. 112 (2004) 983 [gr-qc/0402037] [INSPIRE].

[39] V. Cardoso and J.P.S. Lemos, New instability for rotating black branes and strings, Phys. Lett. B 621 (2005) 219 [hep-th/0412078] [INSPIRE].

[40] V. Cardoso and S. Yoshida, Superradiant instabilities of rotating black branes and strings, JHEP 07 (2005) 009 [hep-th/0502206] [INSPIRE].

[41] S.R. Dolan, Instability of the massive Klein-Gordon field on the Kerr spacetime, Phys. Rev. D 76 (2007) 084001 [arXiv:0705.2880] [INSPIRE].

[42] S. Hod and O. Hod, Comment on 'the extremal black hole bomb', arXiv:0912.2761 [INSPIRE].

[43] H.R. Beyer, On the stability of the massive scalar field in Kerr space-time, J. Math. Phys. 52 (2011) 102502 [arXiv:1105.4956] [INSPIRE].

[44] Y.S. Myung, Instability of rotating black hole in a limited form of $f(R)$ gravity, Phys. Rev. D 84 (2011) 024048 [arXiv:1104.3180] [INSPIRE].

[45] H.R. Beyer, On the stability of the Kerr metric, Commun. Math. Phys. 221 (2001) 659 [astro-ph/0008236] [INSPIRE].

[46] H.R. Beyer, On the stability of the massive scalar field in Kerr space-time, J. Math. Phys. 52 (2011) 102502 [arXiv:1105.4956] [INSPIRE].

[47] S. Hod, On the instability regime of the rotating Kerr spacetime to massive scalar perturbations, Phys. Lett. B 708 (2012) 320 [arXiv:1205.1872] [INSPIRE].

[48] S. Hod, Stability of the extremal Reissner-Nordström black hole to charged scalar perturbations, Phys. Lett. B 713 (2012) 505 [arXiv:1304.6474] [INSPIRE].

[49] J.-P. Lee, Superradiance by mini black holes with mirror, JHEP 01 (2012) 091 [arXiv:1107.5641] [INSPIRE].

[50] J.-P. Lee, Black-hole bombs at the LHC, Mod. Phys. Lett. A 27 (2012) 1250038 [arXiv: 1104.0496] [INSPIRE].

[51] S. Hod, Stability of the extremal Reissner-Nordström black hole to charged scalar perturbations, Phys. Lett. B 713 (2012) 505 [arXiv:1304.6474] [INSPIRE].

[52] S.R. Dolan, Superradiant instabilities of rotating black holes in the time domain, Phys. Rev. D 87 (2013) 124026 [arXiv:1212.1477] [INSPIRE].

[53] H. Witek, V. Cardoso, A. Ishibashi and U. Sperhake, Superradiant instabilities in astrophysical systems, Phys. Rev. D 87 (2013) 043513 [arXiv:1212.0551] [INSPIRE]. 
[54] V. Cardoso, Black hole bombs and explosions: from astrophysics to particle physics, Gen. Rel. Grav. 45 (2013) 2079 [arXiv:1307.0038] [InSPIRE].

[55] J.C. Degollado and C.A.R. Herdeiro, Stationary scalar configurations around extremal charged black holes, Gen. Rel. Grav. 45 (2013) 2483 [arXiv:1303.2392] [InSPIRE].

[56] R. Li, Analytical study of superradiant instability for five-dimensional Kerr-Gödel black hole, Eur. Phys. J. C 73 (2013) 2274 [arXiv:1207.1984] [InSPIRE].

[57] S.-J. Zhang, B. Wang and E. Abdalla, Superradiant instability of extremal brane-world Reissner-Nordström black holes to charged scalar perturbations, arXiv:1306.0932 [INSPIRE].

[58] H. Witek, Black hole dynamics in generic spacetimes, arXiv:1307.1145 [INSPIRE].

[59] Y.S. Myung, Instability of a Kerr black hole in $f(R)$ gravity, Phys. Rev. D 88 (2013) 104017 [arXiv:1309.3346] [INSPIRE].

[60] R. Li, Stability of charged black holes in string theory under charged massive scalar perturbations, Phys. Rev. D 88 (2013) 127901 [arXiv:1310.3587] [INSPIRE].

[61] R. Brito, V. Cardoso and P. Pani, Massive spin-2 fields on black hole spacetimes: instability of the Schwarzschild and Kerr solutions and bounds on the graviton mass, Phys. Rev. D 88 (2013) 023514 [arXiv:1304.6725] [INSPIRE].

[62] S. Hod, Rotating black holes can have short bristles, Phys. Lett. B 739 (2014) 196 [arXiv:1411.2609] [INSPIRE].

[63] H. Okawa, H. Witek and V. Cardoso, Black holes and fundamental fields in numerical relativity: initial data construction and evolution of bound states, Phys. Rev. D 89 (2014) 104032 [arXiv:1401.1548] [INSPIRE].

[64] B. Arderucio, Superradiance: classical, relativistic and quantum aspects, arXiv:1404.3421 [INSPIRE].

[65] M.O.P. Sampaio, C. Herdeiro and M. Wang, Marginal scalar and Proca clouds around Reissner-Nordström black holes, Phys. Rev. D 90 (2014) 064004 [arXiv:1406.3536] [INSPIRE].

[66] S. Hod, Stability of highly-charged Reissner-Nordström black holes to charged scalar perturbations, Phys. Rev. D 91 (2015) 044047 [arXiv: 1504.00009] [INSPIRE].

[67] H.M. Siahaan, Instability of charged massive scalar fields in bound states around Kerr-Sen black holes, Int. J. Mod. Phys. D 24 (2015) 1550102 [arXiv:1506.03957] [INSPIRE].

[68] R. Brito, V. Cardoso and P. Pani, Superradiance, Lect. Notes Phys. 906 (2015) 1 [arXiv: 1501.06570] [INSPIRE].

[69] S. Hod, Quasi-bound states of massive scalar fields in the Kerr black-hole spacetime: beyond the hydrogenic approximation, Phys. Lett. B 749 (2015) 167 [arXiv:1510.05649] [INSPIRE].

[70] J. Wilson-Gerow and A. Ritz, Black hole energy extraction via a stationary scalar analog of the Blandford-Znajek mechanism, Phys. Rev. D 93 (2016) 044043 [arXiv: 1509.06681] [INSPIRE].

[71] Y. Huang and D.-J. Liu, Scalar clouds and the superradiant instability regime of Kerr-Newman black hole, Phys. Rev. D 94 (2016) 064030 [arXiv:1606.08913] [InSPIRE]. 
[72] S. Hod, Analytic treatment of the system of a Kerr-Newman black hole and a charged massive scalar field, Phys. Rev. D 94 (2016) 044036 [arXiv:1609.07146] [InSPIRE].

[73] V. Cardoso, O.J.C. Dias, J.P.S. Lemos and S. Yoshida, The black hole bomb and superradiant instabilities, Phys. Rev. D 70 (2004) 044039

[Erratum ibid. D 70 (2004) 049903] [hep-th/0404096] [INSPIRE].

[74] C.A.R. Herdeiro, J.C. Degollado and H.F. Rúnarsson, Rapid growth of superradiant instabilities for charged black holes in a cavity, Phys. Rev. D 88 (2013) 063003 [arXiv:1305.5513] [INSPIRE].

[75] S. Hod, Analytic treatment of the charged black-hole-mirror bomb in the highly explosive regime, Phys. Rev. D 88 (2013) 064055 [arXiv:1310.6101] [INSPIRE].

[76] S. Hod, Algebraically special resonances of the Kerr-black-hole-mirror bomb, Phys. Rev. D 88 (2013) 124007 [arXiv:1405.1045] [INSPIRE].

[77] S. Hod, Onset of superradiant instabilities in the hydrodynamic vortex model, Phys. Rev. D 90 (2014) 027501 [arXiv: 1405.7702] [INSPIRE].

[78] S. Hod, Onset of superradiant instabilities in the composed Kerr-black-hole-mirror bomb, Phys. Lett. B 736 (2014) 398 [arXiv:1412.6108] [InSPIRE].

[79] S. Hod, Resonance spectra of caged black holes, Eur. Phys. J. C 74 (2014) 3137 [arXiv:1410.4567] [INSPIRE].

[80] J.C. Degollado and C.A.R. Herdeiro, Time evolution of superradiant instabilities for charged black holes in a cavity, Phys. Rev. D 89 (2014) 063005 [arXiv:1312.4579] [InSPIRE].

[81] S.R. Dolan, S. Ponglertsakul and E. Winstanley, Stability of black holes in Einstein-charged scalar field theory in a cavity, Phys. Rev. D 92 (2015) 124047 [arXiv:1507.02156] [INSPIRE].

[82] R. Li and J. Zhao, Superradiant instability of charged scalar field in stringy black hole mirror system, Eur. Phys. J. C 74 (2014) 3051 [arXiv:1403.7279] [INSPIRE].

[83] R. Li and J. Zhao, Numerical study of superradiant instability for charged stringy black hole-mirror system, Phys. Lett. B 740 (2015) 317 [arXiv:1412.1527] [INSPIRE].

[84] R. Li, J. Zhao, X. Wu and Y. Zhang, Scalar clouds in charged stringy black hole-mirror system, Eur. Phys. J. C 75 (2015) 142 [arXiv:1501.07358] [INSPIRE].

[85] R. Li, Y. Tian, H.-b. Zhang and J. Zhao, Time domain analysis of superradiant instability for the charged stringy black hole-mirror system, Phys. Lett. B 750 (2015) 520 [arXiv: 1506.04267] [INSPIRE].

[86] R. Li, J.-K. Zhao and Y.-M. Zhang, Superradiant instability of D-dimensional Reissner-Nordström black hole mirror system, Commun. Theor. Phys. 63 (2015) 569 [arXiv: 1404.6309] [INSPIRE].

[87] N. Sanchis-Gual, J.C. Degollado, P.J. Montero, J.A. Font and C. Herdeiro, Explosion and final state of an unstable Reissner-Nordström black hole, Phys. Rev. Lett. 116 (2016) 141101 [arXiv:1512.05358] [INSPIRE].

[88] S. Ponglertsakul, E. Winstanley and S.R. Dolan, Stability of gravitating charged-scalar solitons in a cavity, Phys. Rev. D 94 (2016) 024031 [arXiv:1604.01132] [INSPIRE]. 
[89] S. Ponglertsakul and E. Winstanley, Effect of scalar field mass on gravitating charged scalar solitons and black holes in a cavity, Phys. Lett. B 764 (2017) 87 [arXiv:1610.00135] [INSPIRE].

[90] S. Hod, The charged black-hole bomb: a lower bound on the charge-to-mass ratio of the explosive scalar field, Phys. Lett. B 755 (2016) 177 [arXiv:1606. 00444] [INSPIRE].

[91] Y. Huang, D.-J. Liu and X.-Z. Li, Superradiant (in)stability of D-dimensional Reissner-Nordström-anti-de Sitter black hole mirror system, arXiv:1606.00100 [INSPIRE].

[92] N. Sanchis-Gual, J.C. Degollado, C. Herdeiro, J.A. Font and P.J. Montero, Dynamical formation of a Reissner-Nordström black hole with scalar hair in a cavity, Phys. Rev. D 94 (2016) 044061 [arXiv:1607.06304] [INSPIRE].

[93] P. Basu, C. Krishnan and P.N.B. Subramanian, Hairy black holes in a box, JHEP 11 (2016) 041 [arXiv:1609.01208] [INSPIRE].

[94] S. Hod, The spinning Kerr-black-hole-mirror bomb: a lower bound on the radius of the reflecting mirror, Phys. Lett. B 761 (2016) 326 [arXiv: 1612.02819] [INSPIRE].

[95] N. Sanchis-Gual, J.C. Degollado, J.A. Font, C. Herdeiro and E. Radu, Dynamical formation of a hairy black hole in a cavity from the decay of unstable solitons, arXiv:1611.02441 [INSPIRE].

[96] R.P. Kerr, Gravitational field of a spinning mass as an example of algebraically special metrics, Phys. Rev. Lett. 11 (1963) 237 [InSPIRE].

[97] S.A. Teukolsky, Rotating black holes: separable wave equations for gravitational and electromagnetic perturbations, Phys. Rev. Lett. 29 (1972) 1114 [INSPIRE].

[98] S.A. Teukolsky, Perturbations of a rotating black hole. 1. Fundamental equations for gravitational electromagnetic and neutrino field perturbations, Astrophys. J. 185 (1973) 635 [INSPIRE].

[99] T. Hartman, W. Song and A. Strominger, Holographic derivation of Kerr-Newman scattering amplitudes for general charge and spin, JHEP 03 (2010) 118 [arXiv:0908.3909] [INSPIRE].

[100] A. Ronveaux, Heun's differential equations, Oxford University Press, Oxford U.K. (1995).

[101] C. Flammer, Spheroidal wave functions, Stanford University Press, Stanford U.S.A. (1957).

[102] P.P. Fiziev, Classes of exact solutions to Regge-Wheeler and Teukolsky equations, arXiv:0902.1277 [INSPIRE].

[103] R.S. Borissov and P.P. Fiziev, Exact solutions of Teukolsky master equation with continuous spectrum, Bulg. J. Phys. 37 (2010) 065 [arXiv:0903.3617] [INSPIRE].

[104] P.P. Fiziev, Teukolsky-Starobinsky identities: a novel derivation and generalizations, Phys. Rev. D 80 (2009) 124001 [arXiv:0906.5108] [InSPIRE].

[105] P.P. Fiziev, Classes of exact solutions to the Teukolsky master equation, Class. Quant. Grav. 27 (2010) 135001 [arXiv:0908.4234] [INSPIRE].

[106] M. Abramowitz and I.A. Stegun, Handbook of mathematical functions, Dover Publications, New York U.S.A. (1970).

[107] S. Hod, Weak cosmic censorship: as strong as ever, Phys. Rev. Lett. 100 (2008) 121101 [arXiv: 0805.3873] [INSPIRE]. 
[108] S. Hod, Asymptotic spectrum of the oblate spin-weighted spheroidal harmonics: a WKB analysis, Phys. Lett. B 717 (2012) 462 [arXiv:1304.0529] [INSPIRE].

[109] H. Yang, D.A. Nichols, F. Zhang, A. Zimmerman, Z. Zhang and Y. Chen, Quasinormal-mode spectrum of Kerr black holes and its geometric interpretation, Phys. Rev. D 86 (2012) 104006 [arXiv:1207.4253] [InSPIRE].

[110] S. Hod, Black-hole perturbation theory: the asymptotic spectrum of the prolate spin-weighted spheroidal harmonics, Phys. Rev. D 87 (2013) 064017 [arXiv:1304.4683] [INSPIRE].

[111] J.M. Bardeen, W.H. Press and S.A. Teukolsky, Rotating black holes: locally nonrotating frames, energy extraction and scalar synchrotron radiation, Astrophys. J. 178 (1972) 347 [INSPIRE].

[112] S. Hod, Eigenvalue spectrum of the spheroidal harmonics: a uniform asymptotic analysis, Phys. Lett. B 746 (2015) 365 [arXiv: 1506.04148] [InSPIRE].

[113] B.F. Schutz and C.M. Will, Black hole normal modes: a semianalytic approach, Astrophys. J. 291 (1985) L33 [INSPIRE].

[114] S. Iyer and C.M. Will, Black hole normal modes: a WKB approach. 1. Foundations and application of a higher order WKB analysis of potential barrier scattering, Phys. Rev. D 35 (1987) 3621 [INSPIRE].

[115] S. Iyer, Black hole normal modes: a WKB approach. 2. Schwarzschild black holes, Phys. Rev. D 35 (1987) 3632 [inSPIRE].

[116] L.E. Simone and C.M. Will, Massive scalar quasinormal modes of Schwarzschild and Kerr black holes, Class. Quant. Grav. 9 (1992) 963 [INSPIRE].

[117] S. Hod, Eigenvalue spectrum of the spheroidal harmonics: a uniform asymptotic analysis, Phys. Lett. B 746 (2015) 365 [arXiv: 1506.04148] [InSPIRE].

[118] S. Hod, The superradiant instability regime of the spinning Kerr black hole, Phys. Lett. B 758 (2016) 181 [arXiv:1606.02306] [InSPIRE]. 